\title{
Free Science from Dogma. or: How the "Pseudogene" Hampered Scientific Progress
}

\author{
Peter Borger ${ }^{1 *}$ and Boris Schmidtgall ${ }^{1}$ \\ ${ }^{1}$ The Independent Research Initiative on Information \& Origins, Studiengemeinschaft “Wort und Wissen", Germany \\ *Corresponding author: Peter Borger, The Independent Research Initiative on Information \& Origins, 79540 Loerrach, Germany.
}

To Cite This Article: Peter Borger, Free Science from Dogma. or: How the "Pseudogene" Hampered Scientific Progress. 2020 - 8(1). AJBSR. MS.ID.001239. DOI: 10.34297/AJBSR.2020.08.001239.

Received: 㘹 March 06, 2020; Published: 些 March 12, 2020

\section{Opinion}

That science and ideologically chosen terminology don't go very well together is the tacit message of a recent article in Nature, which critisized the pseudogene concept [1]. The article argues that the old idea that pseudogenes are functionless is wrong. There is now ample evidence, and more is rapidly accumulating that pseudogenes fulfill a whole series of extremely important biological functions. They play a role in the production of proteins that are important for the embryonic development of living beings. They control the production of proteins by causing small changes in the structure of DNA. What was originally thought of as pseudogenes turn out to be the genes specifying non-coding RNAs, which regulate the activity and production of proteins from thousands of genes.

They are the operation system of the genome. Knowing their functions is knowing a lot more about cancers and diseases. "Pseudo" is greek for "mocking" or "lying. Pseudogenes, which thus translate as "lying genes", were considered to be genes that had lost their function in the course of evolutionary process because of mutations that accumulated and inactivated its original function. They were not really functional genes, but relicts of the evolutionary past. They were considered junk DNA. The term pseudogene became established in literature after a series of truncated genes were found in the genome of the African claw frog (Xenopus laevis) in 1977. These truncated genes looked very similar to the functional $5 \mathrm{~S}$ gene, but they seemed to be corrupted. There were no corresponding proteins present in the cells. In those days, it was concluded that the truncated S5 sequences had to be evolutionary remnants.

It seemed, the truncated genes confirmed the ideas of Susumu Ohno. The term pseudogene became the standard name for almost every sequence of DNA that looked like a shortened or defective gene. Soon, pseudogenes were observed everywhere in the DNA of organisms. They became one of the most important arguments for evolution. After publication of the groundbreaking results of the ENCODE project, which showed that the genome is largely functional [2], scientists now prefer to speak of non-coding DNA instead of junk DNA. Incidentally, this new term is still misleading, as this DNA does contain functional genetic code. It usually codes for RNA molecules, which function as background operational programs that command and co-ordinate activation/deactivation of genes. It is needed for processes in the cell to run smoothly. The term junk DNA is now known as one of the greatest failures of life sciences. The term has left the science stage silently. The pseudogene is currently undergoing the same fate.

Developing technical jargon, nomenclature and terminology is an essential part of science. Particularly in a new discipline, careful selection of terms is of great importance, because these will become the central pillars of scientific theory. An accidental or incorrect choice of terms often causes serious problems afterwards. In the 1960s and 1970s, genetics was still in its infancy. At that time, guiding key terms were chosen to describe molecular processes in the cell. Much of that terminology is still useful today. DNA is still defined as information, and the production of proteins as the decoding of this information.

Terms such as transcription (information transfer from DNA to RNA) or translation (information transfer from RNA to protein) are still relevant and helpful today. Unfortunately, scientists also chose terminology that subsequently proved to be incorrect or misleading. A striking example is the term junk DNA. The term, coined by Japanese-American evolutionary biologist Susumu Ohno, was chosen in the belief that the evolutionary process would have left a lot of "evolutionary rubbish" in the DNA [3]. Ohno argued that evolution proceeded by the duplication and mutation of genes. For 
every functional gene that had occurred naturally during the course of the evolutionary process several broken genes would have to be found in the DNA. The term junk DNA was quickly given to genes that had no apparent function or looked truncated. This led to the term pseudogene.

The article in Nature was remarkable for more than one reason. The title alone was notable. It read: "Overcoming challenges and dogmas to understand the functions of pseudogenes". This raises a number of fundamental questions. Such as: how can the functions of pseudogenes be better understood if they have no function? And what about the peculiar phrase "overcoming dogmas"; do dogmas exist within science? Dogmas are doctrines that are not up for discussion. Dogmas can be obstacles to knowing and understanding; they are anti-scientific. In order to obtain real knowledge, dogmas must therefore be kept out of science. It was the darwinian dogma that provided a circular reasoning: evolution is true, so there must be pseudogenes; we perceive pseudogenes, so evolution is true.

Because of this dogma, for decades virtually no research has been done on junk DNA and pseudogenes. Nature writes that the choice of these terms probably also hindered the development of technologies for research on pseudogenes. "Apart from the fact that the assumption that pseudogenes have no function created a threshold for studying them, it also hindered systematic study due to a lack of robust technologies that would have made it possible to identify biological activities of pseudogenes" [1].

It is clear that the terms junk DNA and pseudogene formed mental thresholds, hurdles to do in depth genetic research. Due to dogmatic, neodarwinian views of the DNA, the search for the functions of pseudogenes was neglected for more than forty years. This hampered the progress of our genetic understanding and severely delayed the development of novel detection technologies in genome biology and medicine. This now is hopefully changing. The article in Nature is an invitation of a novel generation of genome researchers to free science from dogma. From darwinian dogma-that is.

\section{References}

1. Cheetham SW, Faulkner GJ, Dinger ME (2019) Overcoming challenges and dogmas to understand the functions of pseudogenes. Nat Rev Genet pp. 191-201.

2. ENCODE Project Consortium (2012) An integrated encyclopedia of DNA elements in the human genome. Nature 489(7414): 57-74.

3. Ohno S (1972) So much "junk" DNA in our genome. Brookhaven Symp Biol 23: 366-370. 